\title{
Energy Monitoring System for Existing Buildings in Indonesia
}

\author{
Sentagi Sesotya Utami ${ }^{1}$, Faridah $^{1}, N a{ }^{\prime}$ im A. Azizi ${ }^{1}$, Erlin Kencanawati ${ }^{1}$, M. Akbar Tanjung ${ }^{1}$, Balza Achmad $^{1}$ \\ ${ }^{1}$ Department of Nuclear Engineering and Engineering Physics, Universitas Gadjah Mada, Yogyakarta, Indonesia
}

\begin{abstract}
Current studies conducted by JICA, AMPRI and IFC-World Bank, reported that large commercial buildings in Indonesia are not energy and water efficient. One of the cause is the lack of regulation. Meanwhile, effective regulations to reduce energy and water consumption are the concern mostly in a new building to obtain a building permit. This strategy is understandable as retrofitting existing buildings are often more difficult to be implemented, and enforcement is still a major issue in Indonesia. Local governments are currently working on streamlining building permit process as well as developing an online monitoring system for existing buildings. By applying a Building Energy Management System (BEMS) enables to reduce energy consumption up to $15 \%$. An energy monitoring system was designed and installed through this research for Department of Nuclear Engineering and Engineering Physics (DNEEP) building, Faculty of Engineering, Universitas Gadjah Mada. It is a 20 years old two-story building used for educational activities, which consist of classrooms, laboratories, offices and storage spaces. An audit energy was done recently in 2015 where an energy consumption of $261.299,636 \mathrm{kWh} /$ year.m2 was reported. In the existing condition, a power meter is inaccessible and therefore, the only feedback of occupancy behavior in the energy consumption is through the electricity bill. The previous study has shown that building occupants would behave more efficiently if the amount of energy used is notified, and the amount of energy savings are recorded. However, these energy monitoring systems are considered expensive and uniquely tailored for every building. This research aims to design and install a cost effective BEMS based on occupant's satisfactory assessment of the lighting, acoustics, and air conditioning quality. The data will be used as a decision supporting system (DSS) by building management through the use of a GUI. The design of the interface was based on a survey result from the prospective users. Installed energy monitoring system uses a current sensor with an accuracy of $98 \%$ and a precision of 0.04 A while the voltage sensor with an accuracy of $98 \%$ and a precision of $0.58 \mathrm{~V}$. The performance testing shows that the number of web clients influences delay of data transmission. The result of the survey shows that GUI is categorized as fair in design without a significant difference between the perceptions of users with and without survey supervision. KeywordBuilding Energy Management System (BEMS); existing building; Decision Supporting System; sensor; GUI
\end{abstract}

\section{Introduction}

Current studies conducted by JICA, AMPRI and IFCWorld Bank, reported that large commercial buildings in Indonesia are not energy and water efficient. This is caused by ignorance from building owners and managers, architects and engineers, and lack of regulation. While regulations to obtain building permit currently is the most effective way of reducing energy and water consumption, these regulations are a concern mostly in new buildings and are implemented to obtain a building permit. This strategy is understandable as retrofitting existing buildings are often more difficult to be implemented, and enforcement is still a major issue in Indonesia. However, as the local governments of Jakarta, Bandung, Surabaya, and Makassar are currently working on streamlining building permit process as well as developing an online monitoring system for existing buildings, the next step is to regulate the techniques for energy and water efficiency improvements. Its implementation is far from ideal as there are no standardized monitoring system in most, if not all, existing buildings.

By applying a building energy monitoring system (BEMS) enables to reduce energy consumption up to 5$15 \%$ [1]. The building owner, building managers, and professionals will be able to operate and retrofit existing buildings to become more efficient in their energy consumption given the data obtained from the monitoring system. It is a significant benefit since the cost of energy is becoming more expensive, in line with the Government 
policy to gradually lift the subsidy. The previous study has shown that building occupants would behave more efficiently if the amount of energy used is notified, and the amount of energy savings are recorded [2].

João Gil Josué et al. developed a BEMS for domestic buildings as a home energy management system [3]. Radio wave and microcontroller PIC18F2420 supported the data transmission system. The maximum line of sight between the data acquisition system and the data interface is 250 meters, sending graphics of voltage, current, power factor, active power and energy data.

The concept of the Internet of Things (IoT) was first used in 1999 by Kevin Ashton to the defined concept of computer and machines with sensors, which connect to the Internet to report status and accept control commands [4]. This concept provides the ability to develop an energy monitoring system supporting the complexity of a building usage. Web-based BEMS has already been developed using the internet from a Wi-Fi network as the data transmission system [5]. Since data are transmitted from the data acquisition system to the web server through the internet, the positioning of the data acquisition system and $\mathrm{Wi}-\mathrm{Fi}$ router determine the data transmission quality indicated by time delay. A database is then provided at the end-user after the data is sent to the web server.

S. Tepić et al., developed a smart data transmission system for BEMS using an efficient software framework [6]. The efficient framework consisted of a message protocol based on HTTP application layer and JSON data package and Internet Protocol (IP) to increase flexibility in the use of physical layers that connect sensors with the entire wireless network. However, these BEMS are considered expensive and uniquely tailored for every building.

Existing buildings in Indonesia are mostly found without as-built drawing documents including information of the building electrical wiring and building service utilities. This is due to the lack of regulations and its reinforcement in the past, during the design, construction until the building commissioning phase. Therefore, to achieve the most effective BEMS implemented in existing buildings, a thorough investigation of the building condition and proper functionality of its premises, is required. As a consequence, the BEMS should be tailored to a unique sensor device and networks, data acquisition and transmission system, and customized the interface for data analysis by the BEMS user (building management) for each building.

This paper implemented the above idea to BEMS development in the Department of Nuclear Engineering and Engineering Physics (DNEEP) building, Faculty of Engineering, Universitas Gadjah Mada (UGM). It is a 20 years old two stories building that consists of classrooms, laboratories, offices and storage spaces. An audit energy was done recently in 2015 where an energy consumption of $261.299,636 \mathrm{kWh} /$ year.m2 was reported. In the existing condition, a power meter is inaccessible and therefore, the only feedback of occupancy behavior in the energy consumption is through the electricity bill. This research aims to design and install an effective BEMS in the DNEEP building, based on occupant's satisfactory assessment of the lighting, acoustics, and air conditioning quality. The data will be used as a decision supporting system (DSS) by building management through the use of a GUI. The design of the interface was based on a survey result from the prospective users.

\section{Method}

There are mainly three important components in the BEMS, which leads to utilization of several steps and techniques during the development process (Fig. 1). Three components are the sensors, the data transmission system, and the interface as a DSS. It involves two functions, which are data acquisition and processing, and real-time monitoring based on Internet of Things (IoT).

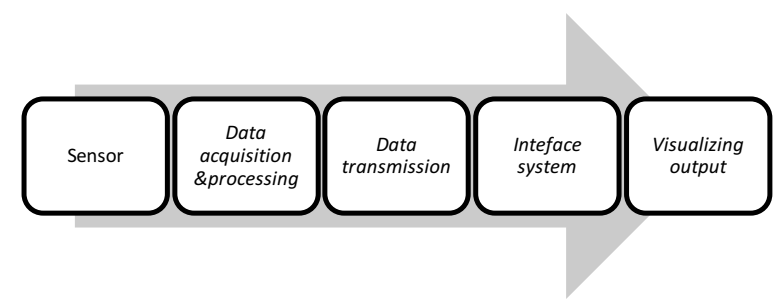

Fig. 1. Steps of the development process.

The system sensor is designed to measure the electricity load by using an electrical current sensor SCT 013-000 to sense the electrical current $i(t)$ and sensing the voltage $v(t)$ using sensor ZMPT101B. Sensors are tested using the input and output correlation used as the calibration factor. The correlation value depends on the condition of the electrical wires of the building's utilities. The calibration factor will be used by the microcontroller to process data and produce measured values, which are accurate and precise.

Using the measured $i(t)$ and $v(t)$, the amount of energy consumption is generated by the BEMS based on the average power-usage. The electrical current and voltage passing through the system sensor are defined as (Fig. 2):

$$
\begin{aligned}
& i(t)=I_{m} \cos \left(\omega t+\theta_{i}\right) \\
& v(t)=V_{m} \cos \left(\omega t+\theta_{V}\right)
\end{aligned}
$$

Meanwhile, the average power of one cycle with period $T$ is given by:

$$
P=\frac{1}{T} \int_{0}^{T} v(t) i(t) d t=V_{r m s} I_{r m s} \cos \left(\theta_{V}-\theta_{i}\right)
$$

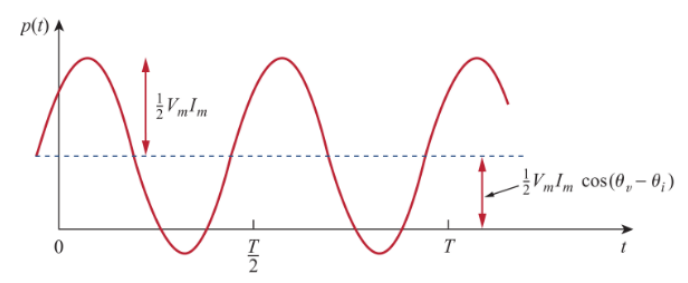


Fig. 2. Instantaneous power in circuits [7].

It seems as if $V_{r m s} I_{r m s}$ is already providing the power value needed. However, this value is not yet the real average power since there is still the factor of $\cos \left(\theta_{V}-\right.$ $\theta_{i}$ ) from the Equation 3 above. This unreal average power is known as appearance power [8]. Ratio between the appearance power to the average power is known as the power factor shown in equation

$$
p f=\frac{P}{V_{r m s} I_{r m s}}=\cos \left(\theta_{V}-\theta_{i}\right)
$$

The energy used, $W$, that will be reported is calculated from the average power, $P$, being sampled for each period by $\Delta t=1 / 50 \mathrm{~s}$, or can be written as,

$$
W=P . \Delta t
$$

Output data from the sensors system are stored in the data logger, which these parts stand as the data acquisition system. Data can also be sent to a server using the IoT supporting system (Fig. 3). There are three characteristics of IoT, which are the perception layer, network layer, and application layer [9]. Perception layer is the layer that interacts with all the items outside the IoT system, which in this case are the sensors used to obtain the information. The network layer is also known as the transportation layer due to its function in transmitting data from the perception layer to the application layer. Shortly speaking, this layer has a function to transmit the data through the internet from microcontroller to the web server and from the web server to the client. Application layer plays a role in processing and analysing data obtained from the perception layer in the form of a GUI, which can be accessed by the users to get information about the energy consumption.

The network layer of the BEMS developed in this research refers to web application design with the RESTful Web Service system. It implements the architectural concept of Representational State Transfer (REST). Given the principle of RESTful Web Service, the data transmission protocol is using HTTP Protocol. Aside to the use of HTTP Protocol, this system also uses JSON as standard data format, which is understandable to the web client and web server. It also identifies each resource using Uniform Resources Identifier (URI) [10]. The performance of this network layer is indicated by the time delay.

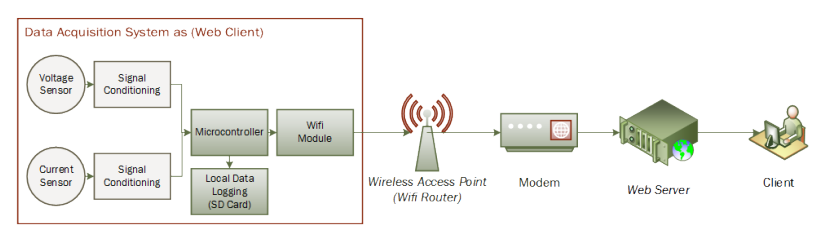

Fig. 3. Block Diagram of energy monitoring system.

The application layer refers to the design of Graphical User Interface (GUI) based on the concept of designing an Energy Monitoring and Targeting (EM\&T). Slightly different with Building Energy Management System
(BEMS) that provides a real-time control of the building utilities to perform its functions in terms of safety, efficiency, and comfort, EM\&T only monitors the energy consumption by collecting data and analyzing the history. There are two main functions of EM\&T. By monitoring the energy consumption and compare the energy usage of previous time or standards of typical buildings, the building user can control the energy use with EM\&T. The second function is the ability to increase energy efficiency by defining an energy consumption target for the next period of analysis [11]. There are four monitoring processes in the BEMS that are considered during the GUI design, which are:

1. Data collection, a process in collecting data on the energy consumption of the area, monitored and also other relevant data.

2. Data Analysis, data received by the computer are examined of any errors and check whether the data are saved correctly for the analysis. The analysis is determined by the system requirement.

3. Reporting, a stage where the energy consumption report is produced and being compared with the target.

4. Action, implementation of the report by the building management. This action is needed to effectively reduce the energy consumption where the previous consumption was reported by the BEMS.

The client is able to monitor the energy consumption by accessing the web address using a common web browser. Every 15 minutes the electricity power usage, $\mathrm{P}$ and energy usage, $\mathrm{W}$ are sent from the sensors. An interface in the application layer is designed to provide easiness for the user in accessing information from the monitoring system. Data are presented in graphics and charts, which is then used as the statistical analysis in the energy consumption report. Several indoor quality parameters that are strongly related to the building energy consumptions, which are lighting, acoustics, and air conditioning quality, are also displayed and reported. This interface was design based on the clients need in order to create an interesting, informative and easy-used web interface. A survey was conducted to ensure the user's needs were already accommodated by the interface designed.

\section{Results and Discussions}

\subsection{Sensors and Data Processing}

\subsubsection{Electricity Current Sensor System.}

The sensor for electrical current is using SCT 013-000 with a microcontroller as a data processing tool shown in Figure 4.

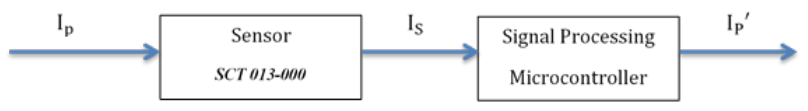

Fig. 4. The sensing method of the current sensor. 
The SCT 013-000 sensor has a range of input AC current $\left(I_{p}\right)$ of $0-100$ ampere $(A)$ and a range of output $A C$ current $\left(\mathrm{I}_{\mathrm{S}}\right)$ of $0-50 \mathrm{~mA}$. The transduction process uses the principle of induction as in an ordinary transformer. Electricity current measured that flows through the wire will cause magnetic field at the magnetic core and produced the output AC current $\left(\mathrm{I}_{\mathrm{S}}\right)$ at the secondary coil. Zener diode in the internal sensor has a function as a circuit protector to avoid extremely high voltage due to the change in electrical current during transient conditions. Fig. 5 describes the relation between $I_{s}$ and $I_{p}$ or commonly known as a transfer function. The sensor transfer function is

$$
\mathrm{I}_{\mathrm{S}}=0.0005 \mathrm{I}_{\mathrm{p}}
$$

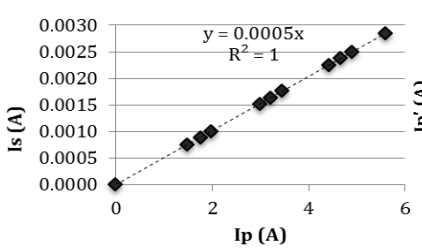

(a)

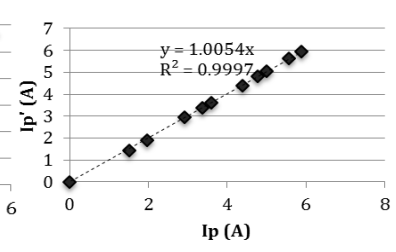

(b)
Fig. 5. Transfer function of the sensor SCT-000, and (b) and with the entire system (current sensor and microcontroller)

This relation is then used as the calibration factor of the microcontroller Arduino with an output current measured, $\mathrm{I}_{\mathrm{p}}{ }^{\prime}$, which is similar to the actual electrical current. Meanwhile, Fig.6 describes how the system works, the relation between $I_{p}$ and $I_{S}$. The accuracy and precession of the sensor system are $98 \%$ and $0.04 \mathrm{~A}$, respectively.

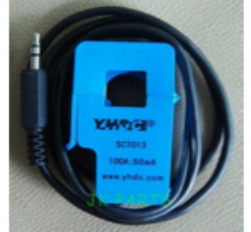

(a)

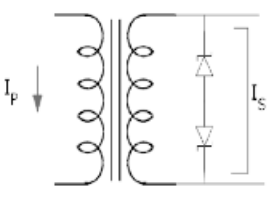

(b)
Fig. 6. (a) Electricity current Sensor SCT-000, and (b) diagram of how the current sensor works

\subsubsection{Electricity Voltage Sensor System.}

The sensor system for measuring the electrical voltage used in this BEMS consists of a voltage sensor ZMPT101B and a microcontroller to process the data as described in the block diagram in Fig. 7.

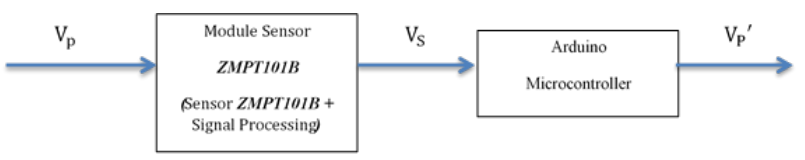

Fig. 7. The sensing method of the voltage sensor.

The ZMPT101B voltage sensor also works using the principle of a simple transformer. The measured or input voltage $\left(V_{p}\right)$ will cause electric current $\left(I_{P}\right)$ and induce the output electric current $\left(\mathrm{I}_{\mathrm{S}}\right)$, which will flow with a resistance of $R$, creating the output voltage $\left(V_{S}\right)$. Fig. 8 shows the relation between $V_{p}$ and $V_{S}$. The transfer function of the voltage sensor is given by,

$$
\mathrm{V}_{\mathrm{S}}=0.0035 \mathrm{~V}_{\mathrm{p}}
$$

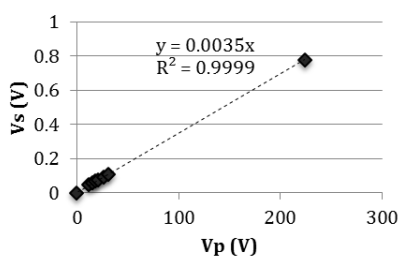

(a)

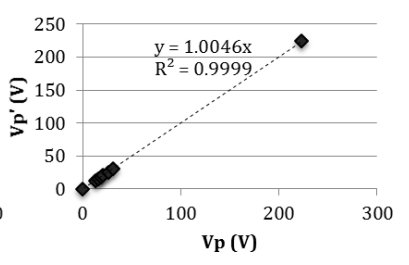

(b)
Fig. 8. (a) Transfer function of the sensor ZMPT101B, and (b) and with the entire system (voltage sensor and microcontroller)

Similar to the current sensor, this transfer function is used as the calibration factor in the microcontroller to obtain the output voltage of the system $V_{p}{ }^{\prime}$, which has the same value as the input voltage $\left(\mathrm{V}_{\mathrm{p}}\right)$. Meanwhile, Fig. 9 describes how the system works and the relation between $\mathrm{V}_{\mathrm{p}}{ }^{\prime}$ and $\mathrm{V}_{\mathrm{p}}$. The accuracy and precession of the sensor system are $98 \%$ and $0.58 \mathrm{~V}$, respectively.
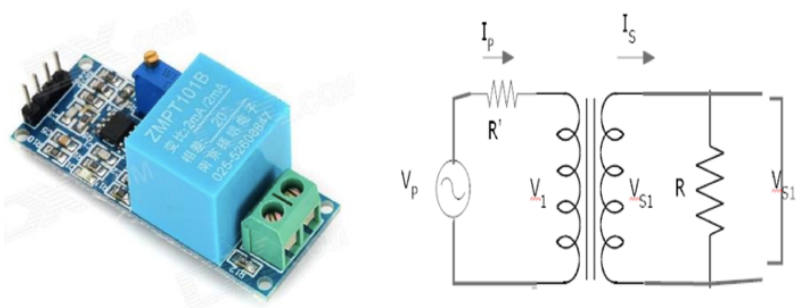

Fig. 9. (a) Electricity voltage sensor ZMPT101B, and (b) diagram of how the voltage sensor works.

\subsubsection{Sensor positioning and installation}

The positioning of the sensors is based on the analysis of the electrical wiring diagram of DTNTF building. As in the majority of an existing building in Indonesia, the asbuilt drawing of the case study also does not exist. Therefore no electrical wiring diagram is available. An on-site survey is required where every wire is traced to get the electrical wiring diagram of the electrical lighting and $\mathrm{AC}$ of DTNTF building. Ideally, the wiring of each building equipment or utilities should be traceable in order to have the energy consumption data of this equipment. If this information is available, the building manager will be able to come up with strategies for reducing the energy consumption. The closest approach in tracing the equipment's wiring is by grouping equipment into zones, which is the first attempt of this BEMS designed.

\subsection{Data Transmission}

Beside as a data processor in the sensors system, the microcontroller Arduino also acts as a web client. As a 
web client, Arduino sends data from the sensor to the web server. The data consists of $V_{r m s}, I_{r m s}$, power factor, average power, and the energy consumption. The complete sensor system with the Arduino web client is a box sensor. Each box sensor consists of four packages of data sensor and therefore enables to measure four different electricity wires. The data transmission algorithm within the Arduino is using HTTP method followed by URL and data packet. This data packet consists of measured data, sensor identifier, and zone identifier. All the data within the Arduino are sent periodically at certain intervals. In this BEMS, data are sent every 15 minutes.

Web server is used to save the web application at a certain domain (Fig. 10). The web server in the web application is using the RESTful Web Service system, which creates HTTP Router. These routes were written in Hypertext Preprocessor (PHP) coding language and can be accessed by the web client by using URL and HTTP request method. Every HTTP Router has a different response and function. With this HTTP Router, the web client is able to send data to the database and access the resources that are kept in the web server or database system.

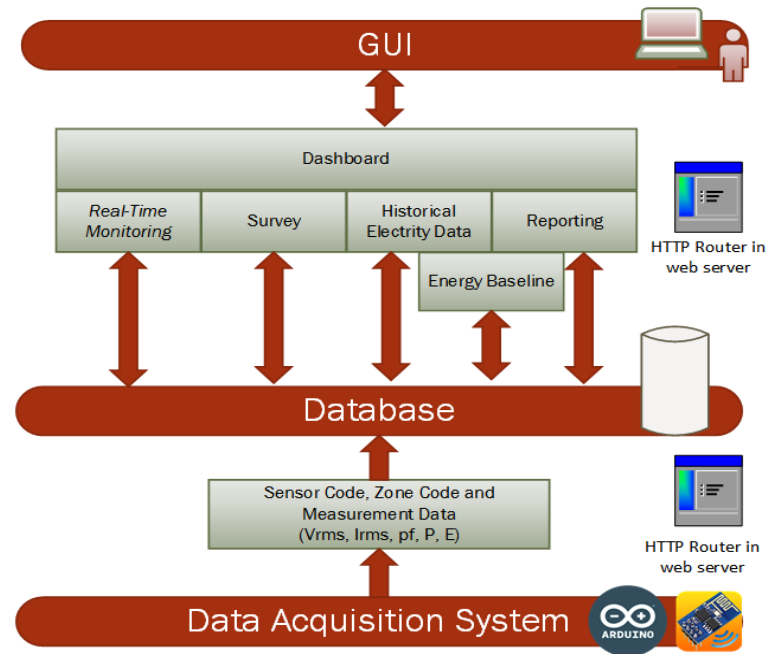

Fig. 10. Structure and function design of HTTP Router in web server.

The figure illustrates the HTTP Router in the web server of the BEMS. The main function of the HTTP Router can be categorized as two paths, which are the path from GUI and the path from the microcontroller Arduino as the web client. In the GUI path, there is an HTTP Router that can be used by the user to access pages of the dashboard as the home page of the BEMS GUI. From the dashboard pages, users can access real-time monitoring pages, survey, historical data and report of the energy consumption. In each page, the HTTP Router is also being functioned to process and display the data from the database as what is needed for each page. In the Arduino path as the web client, there is an HTTP Router that serves to receive the data measured being sent by the Arduino and save the data into the database.
The result of testing the time delay of data transmission system by varying the number of web client is shown in Fig. 11. Web clients used in the testing of the data transmission system are Arduino and users who access GUI by using a web browser. The differences between those web clients are Arduino as web clients send data to a web server however users request data from the web server to display the web page. The average delay for each variation of web clients is $17 \mathrm{~ms}$ for 1 web client, 64 $\mathrm{ms}$ for 10 web clients, $253 \mathrm{~ms}$ for 50 web clients and 566 for 100 web clients. Based on the result, energy monitoring system can be developed on a larger scale with the consequence of long time delay.

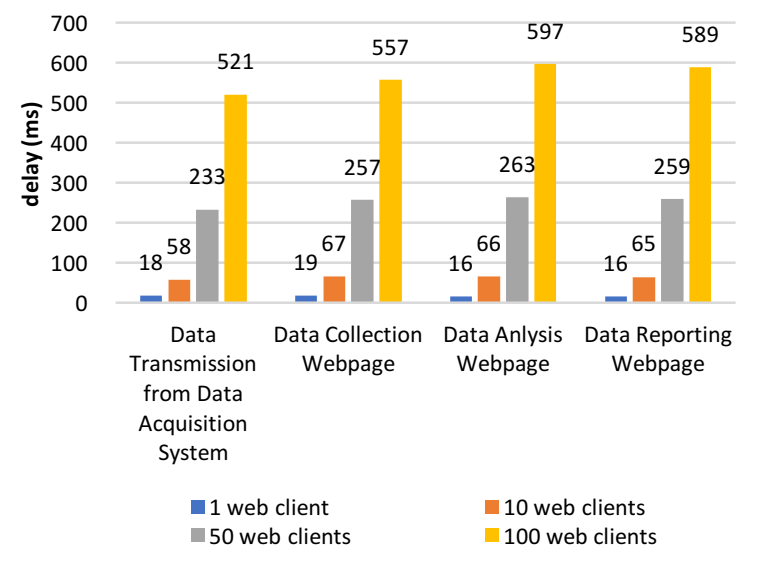

Fig. 11. Performance testing of energy monitoring system by varying the number of web clients.

\subsection{Interface}

The concept of EM\&T is used to develop the GUI of this system as mentioned earlier. The dashboard of this BEMS's GUI is shown in Fig. 12. This version is still in Bahasa Indonesia. The monitoring system is part of the BEMS, which integrates with the monitoring of the lighting condition, temperature, humidity, and volume of $\mathrm{CO} 2$ of the building.

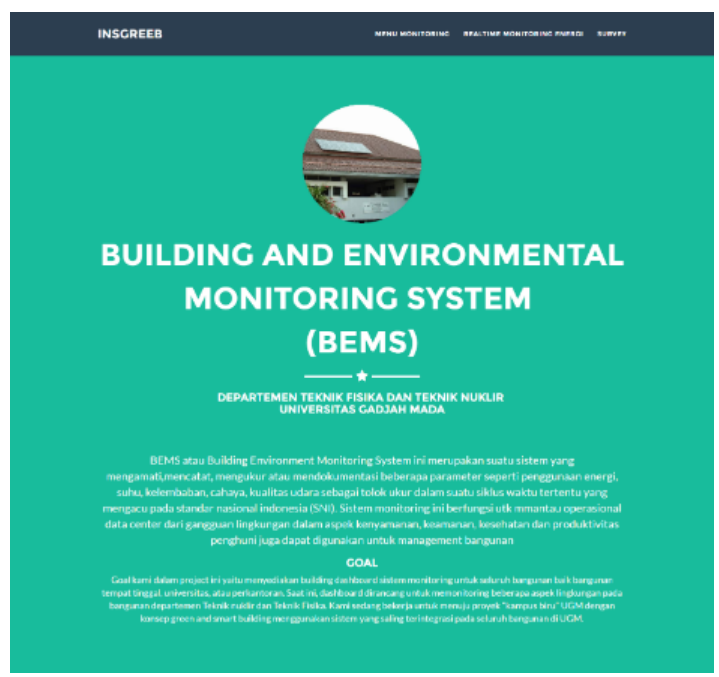

Fig. 12. Overview of BEMS, navigation of the GUI and the location of the installation of the monitoring system. 


\subsubsection{Data Collection}

A Real-time monitoring inside the dashboard is display as graphics that show the energy consumption in real time starting from 00.00 am each day as shown in Fig. 13. Data of real-time are within the interval of 15 minutes complying with the time interval of data transmission from the Arduino, web client.

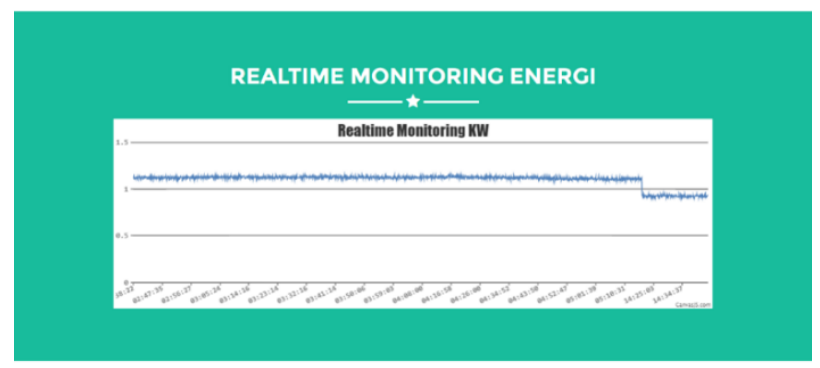

Fig. 13. Chart of real-time energy monitoring.

\subsubsection{Data Analysis}

In the EM\&T concept, data are display in pages of data history to meet the user needs, which is the building management. Data history enables to monitor the daily energy consumption and compares with the baseline condition (see Fig. 14). Energy baseline is the energy usage threshold defined by the building management. The threshold of power usage from the energy baseline from time to time is known as the power baseline. Both data of the energy and power baseline are provided through audit energy of the existing condition.

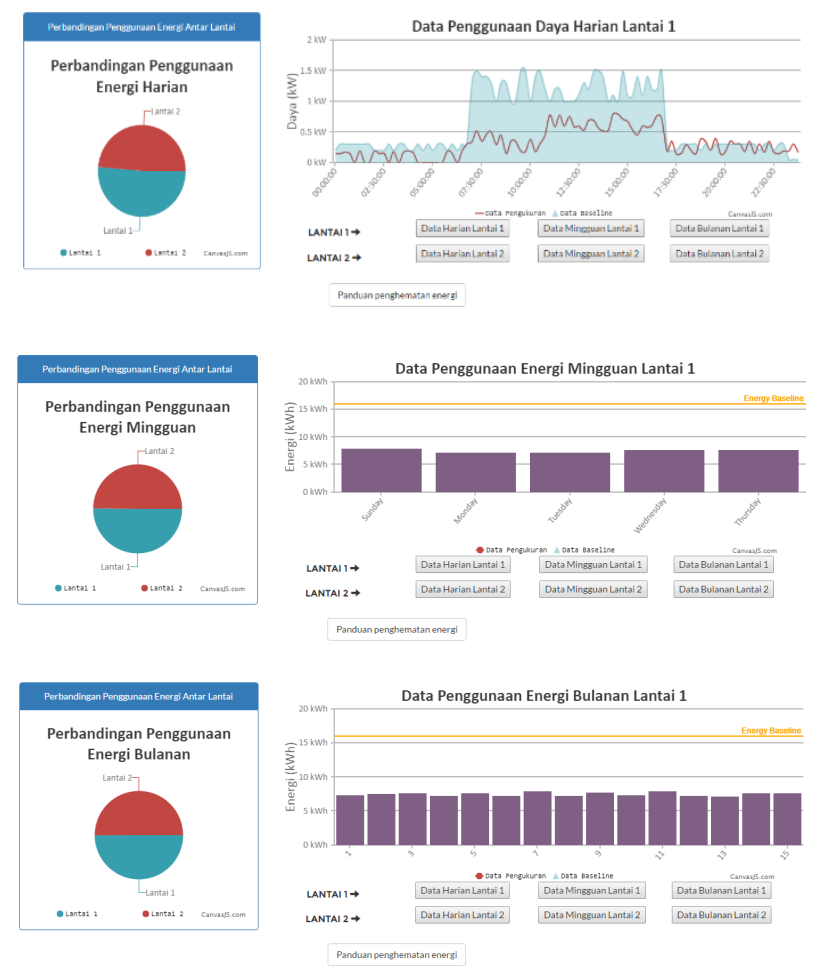

Fig. 14. Chart of energy usage analysis with energy baseline.
Under the graphic that displays the energy consumption, some energy-saving strategies are provided in the form of a slideshow, in Bahasa Indonesia, to help the user in understanding technical and non-technical energy-saving strategies (see Fig. 15).

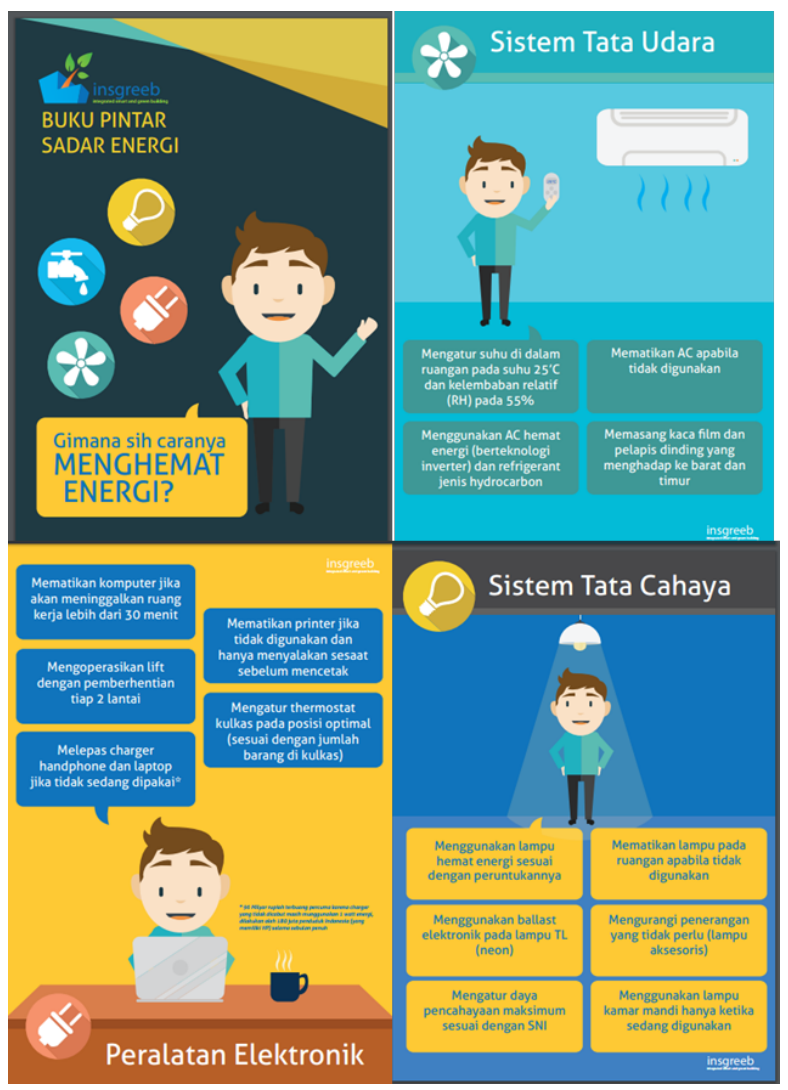

Fig. 15. Energy saving tips to help client carry out energy management (in Bahasa Indonesia).

\subsubsection{Data Reporting}

Annual report of the energy consumption is also provided in the GUI of the energy monitoring system (see Fig. 16). The annual report is chosen since the energy use cycle is for a year. Energy consumption is influenced by the surrounding climate of the building and activities of its surrounding, which is usually observed as one year cycle.

The final step of the EM\&T concept is the action which is not yet implemented in this BEMS since it depends on to the building management policy given the energy consumption data. The use of GUI based on the survey conducted by building user has shown that the design of the GUI is categorized as fair, with a score of 3.71 points out of 5 for respondents that have gone through some supervision before filling out the survey. There is no significant difference in the GUI perception of two different respondents group, with and without survey supervision. 


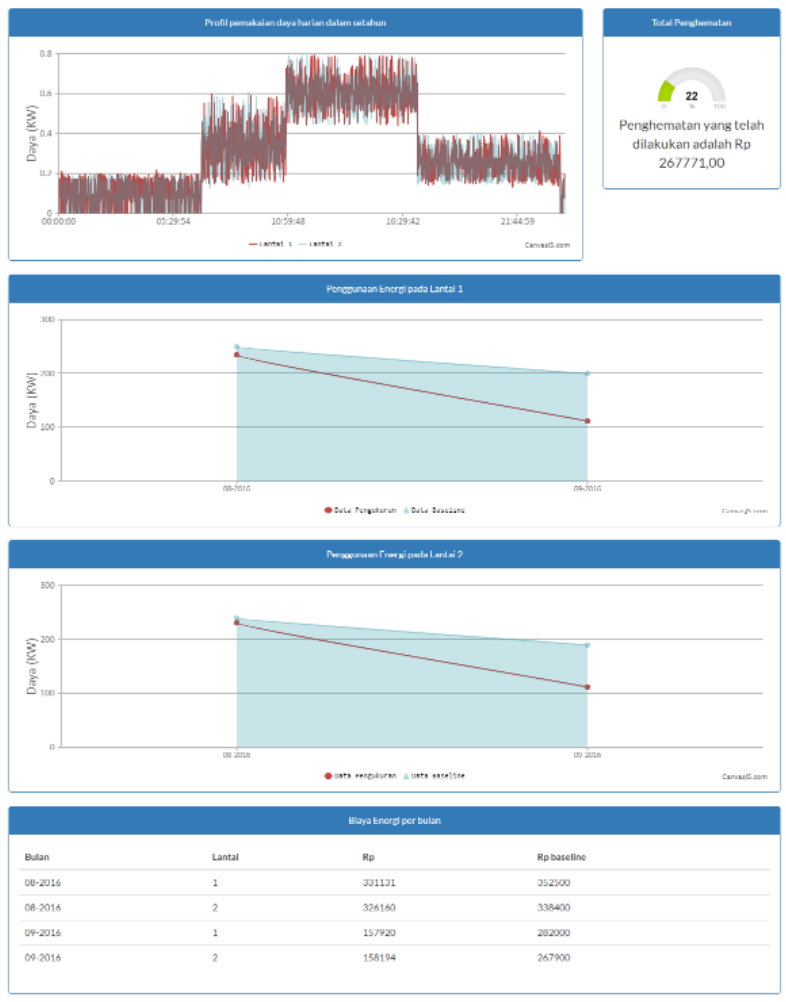

Fig. 16. Annual reporting of energy monitoring.

\section{Conclusion}

This research has shown that a building energy management system (BEMS) for every existing building is uniquely designed and specifically tailored due to the complexity of the existing wiring system, building zonation, and building utility or equipment that are being operated. It requires several steps from the characterization of the sensors, reliability test of the data transmission system and a survey to develop a humanfriendly interface as an efficient DSS for the building management.

Based on test results, the current sensor has an accuracy of $98 \%$ and a precision of 0.04 A while the voltage sensor has an accuracy of $98 \%$ and a precision of $0.58 \mathrm{~V}$. The average delay of data transmission testing for each variation of web clients are $17 \mathrm{~ms}$ for one web client, $64 \mathrm{~ms}$ for $10 \mathrm{web}$ clients, $253 \mathrm{~ms}$ for $50 \mathrm{web}$ clients and 566 for 100 web clients. Based on the result data transmission testing, energy monitoring system can be developed on a larger scale with the consequences of long time delay. The results of a user survey show that the design of the GUI is categorized as fair, without a significant difference between the perceptions of users with and without survey supervision.

The results also provide important information for further improvement of the system as more sensors will be implemented for the entire building and can be used as a benchmark for other similar building. In other words, the final product presented in this paper is a bone of a BEMS for the DTNTF building. The target of the building energy efficiency can be obtained after several years of data collection. Approximately $10 \%$ of the energy consumptions for several zones in the building area is provided as a baseline, and the data complies with the audit energy data gathered in 2015.

\section{References}

[1] S. Darby and others, The effectiveness of feedback on energy consumption, Rev. DEFRA Lit. Metering Billing Direct Disp 486, 2006, (2006)

[2] W. Kempton, J.M. Darley, P.C. Stern, Am. Psychol 47, 1213-1223 (1992)

[3] J.G. Josue, J.M. Pina, M.V. Neves, Doctoral Conference on Computing Electrical and Industrial Systems 2011, 437-444 (2011)

[4] D. Norris, The Internet of Things: Do-It-Yourself at Home Projects for Arduino, Raspberry $\mathrm{Pi}$ and BeagleBone Black (McGraw-Hill Education, New York, 2015)

[5] Y. Qu, H. Wang, S.M. Lund, H.D. Chiang, T. Wang, Design and implementation of a Web-based Energy Management Application for smart buildings, 2013 IEEE Electrical Power Energy Conference (EPEC), $1-6(2013)$

[6] S. Tepić, P. Pejić, J. Domšić, H. Mihaldinec, H. Džapo, 2015 38th International Convention on Information and Communication Technology, Electronics and Microelectronics (MIPRO), 143148 (2015)

[7] C. Alexander, M. Sadiku, Fundamentals of Electric Circuit Analysis (McGraw-Hill Higher Education, Columbus, 2012)

[8] W.H. Hayt, J.E. Kemmerly, Engineering Circuit Analysis (Mc Graw Hill Higher Education, Columbus, 1971)

[9] X. Yang, Z. Li, Z. Geng, H. Zhang, Internet of Things, 388-393 (Springer, Berlin, 2012)

[10] S. Abeysinghe, Restful PHP web services: Learn the Basic Architectural Concepts and Steps Through Examples of Consuming and Creating Restful Web Services in PHP (Packt Publishing, Birmingham, 2008)

[11] P. Warburton, K. J. Butcher, Chartered Institution of Building Services Engineers, Eds, Building control system, 2. ed, London: CIBSE, (2009) 